\title{
Calculated model of wedge-shaped sliding supports in turbulent friction regime
}

\author{
Akhverdiev Kamil Samedovich \\ «Rostov state transport university» (RSTU), \\ 2, Rostovskogo Strelkovogo Polka Narodnogo Opolcheniya \\ sq., Rostov-on-Don, 344038, Russia \\ vm_2@kaf.rgups.ru
}

\section{Lagunova Elena Olegovna}

«Rostov state transport university» (RSTU),

2, Rostovskogo Strelkovogo Polka Narodnogo Opolcheniya

sq., Rostov-on-Don, 344038, Russia

lagunova@rambler.ru

\begin{abstract}
The solution of the problem has been found on the basis of a system of equations describing the motion of an incompressible liquid electrically conductive lubricant for the case of a "thin layer", the continuity equation and the expression for the dissipation rate of mechanical energies to determine the function caused by the melt of the guide surface coated with a melt of a fusible coating. Asymptotic solution of a system of differential equations taking into account the boundary conditions on the surface of the guide, slider and contour are found in the form of series in powers of the small parameter $K$, caused by the melt and the rate of dissipation of mechanical energy. To determine the velocity and pressure fields in the lubricating and molten layer, a precise self-similar solution for the zero and first approximations is found. As a result of finding the exact self-similar solution, the value of the function, caused by the melt of the guide is found.
\end{abstract}

The influence of the following parameters has been estimated: caused by the presence of an electric field, Hartmann number, caused by the melt and the rate of dissipation of mechanical energy on the main operating characteristics of the thrust sliding bearing (bearing capacity and frictional force).

Difference from the existing design models of sliding supports operating on the melt of a low-melting coating is the consideration of a whole range of variable factors allowing increasing the accuracy of models approximating to the real needs of practice.

The results of numerical analysis show that the design models of thrust sliding bearings have been significantly refined as a result of additional simultaneous consideration in their development of the dependence on the hydrodynamic pressure and temperature of such important factors as electrical conductivity, as well as the effects of magnetic induction and electric field strength.

Keywords-melt; low-melting coating; wedge-shaped support; electrically conductive lubricant; energy dissipation rate

\author{
Kolesnikov Igor Vladimirovich \\ «Rostov state transport university» (RSTU), \\ 2, Rostovskogo Strelkovogo Polka Narodnogo Opolcheniya \\ sq., Rostov-on-Don, 344038, Russia \\ oooedt@rambler.ru \\ Mukutadze Murman Alexsandrovich \\ «Rostov state transport university» (RSTU), \\ 2, Rostovskogo Strelkovogo Polka Narodnogo Opolcheniya \\ sq., Rostov-on-Don, 344038, Russia \\ murman1963@yandex.ru
}

\section{INTRODUCTION}

The main friction knot of modern machines is sliding bearings, lubricated with liquid lubricants. For modern engineering practice, sliding bearings are designed taking into account the increase in static and dynamic loads. At high load and speed regimes, the use of mineral oils as lubricants causes certain difficulties. They are realized in the form of scratching and seizures of I and II kind. To avoid these drawbacks, especially when leaking or evaporation of the lubricant from the bearing, a backup lubricant may be the coating of the bearing surface of a low-melting metal bearing sleeve.

Lubrication with liquid metals is used at temperatures at which conventional lubricating media undergo irreversible physicochemical properties. The advantage of lubrication with the melt is that the lubricant is formed in the contact area where it is necessary. Melting delivers a sufficient amount of lubricant material to the friction zone, there are no mechanical and structural difficulties associated with its feeding. Lubrication with a melt was studied in many applied problems, in particular, in the processes of forming and cutting metals [1-12]. A large number of works have been devoted to hydrodynamic calculation of sliding bearings in the absence of a lubricant and taking into account the dependence of the viscosity of the lubricant on pressure. A significant drawback of the friction pair operating on melt lubrication is a low loadbearing capacity. In addition, the lubrication process with the lubricant is not self-sustaining.

The works are devoted to theoretical analysis of the work of radial and thrust sliding bearings in the presence of a lubricant of a tribological system caused by the melt of a surface covered with a low-melting metallic melt when taking into account the dependence of the viscosity of the lubricant on pressure [13-16].

A number of works are dedicated to the development of the design model of radial and thrust sliding bearings, taking 
into account the rheological properties of micropolar, viscoelastic lubricant, taking into account the dependence of the viscosity of the lubricant on the pressure and melt of the fusible coating [17-24].

In this paper, the solution of the problem is given for the case when the lubricant has the properties of the electrically conductive lubricant.

Thus, the development of theoretical bases for the calculation of tribosystems lubricated by a metallic melt, taking into account the rheological properties of an electrically conductive lubricant under the condition of the action of an electromagnetic field, the justification of a dynamics and lubrication model that outstrips the real processes occurring in the lubricating layer, and the creation of algorithms and software for solving practical tasks, increasing the reliability of machines and mechanisms determines the relevance of this article.

\section{TASK SETTING}

The turbulent flow of a viscous electrically conductive lubricant between an inclined slider and a guide is considered. It is assumed that the surfaces of the slider and the guide are separated by a layer of a lubricant having electrically conductive properties, the slider is stationary, and the guide made of the material with a low melting point moves toward the narrowing of the gap at a speed $u^{*}$ (Fig. 1).

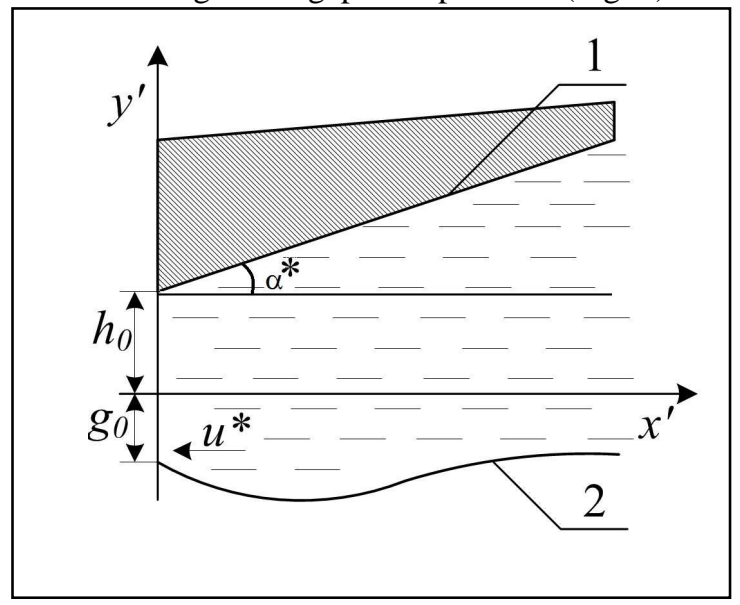

Fig. 1. Working scheme

The dependence of the viscosity and electrical conductivity of a liquid lubricant is given in the form:

$$
\mu^{\prime}=e^{\alpha^{\prime} p^{\prime}-\beta^{\prime} T^{\prime}}, \quad \sigma^{\prime}=e^{\alpha^{\prime} p^{\prime}-\beta^{\prime} T^{\prime}}
$$

where $\mu^{\prime}$ is the coefficient of a dynamic viscosity of the lubricant; $p^{\prime}$ is hydrodynamic pressure in the lubricating layer; $\alpha^{\prime}, \beta^{\prime}$ are experimental constant values; $\sigma^{\prime}$ is electric conductivity of the lubricating material, $\mathrm{T}$ is temperature in lubricating layer.

The conditions for the motion of an infinitely wide slider are considered under the following assumptions:
1 The liquid medium is a viscous incompressible fluid.

2 All the heat released in the lubricating film goes to the melting surface of the material of the guide.

3 The effect of turbulence can be reflected using the coefficient $j>1$, to which the viscosity should be multiplied in order to obtain the effective viscosity value. In addition, it is assumed that this coefficient can be expressed as the following function of the Reynolds number $j=0,0139 \mathrm{Re}^{0,657}$, where $\operatorname{Re}=\rho u^{*} h_{0}^{2} / \mu_{0} l$ is the Reynolds number, $\mu_{0}$ is the dynamic viscosity, $h_{0}$ is the film thickness in the initial section, $\rho$ is density, $u^{*}$ is the movement speed slider, $l$ is the length of the fixed working surface of the bearing (slider).

\section{INITIAL EQUATIONS AND BOUNDARY CONDITIONS}

As initial equations, we consider the dimensionless equations of motion of a lubricant possessing electrically conductive properties for the case of a "thin layer" with allowance for (1), the continuity equation, and the formula for the rate of dissipation of mechanical energy for determining the function $\Phi(x)$, caused by the molten contour of the surface of the guide:

$$
\begin{gathered}
\frac{\partial^{2} v}{\partial y^{2}}=\frac{1}{j e^{\alpha p-\beta T}} \frac{d p}{d x}+N v-A, \quad \frac{\partial u}{\partial y}+\frac{\partial v}{\partial x}=0, \\
\frac{d \Phi(x)}{d x}=K \int_{-\Phi(x)}^{h(x)}\left(\frac{\partial v}{\partial y}\right)^{2} d y
\end{gathered}
$$

where $u, v$ are components of the velocity vector of the lubricating medium; $N=\frac{\sigma_{0} B h_{0}^{2}}{\mu_{0}}$ is Hartmann number, $\bar{B}=\left\{0 ; B_{y} ; 0\right\}$ is magnetic induction vector, $\bar{E}=\left\{0 ; 0 ; E_{z}\right\}$ is the electric field strength vector, $B_{y}$ is the component of the magnetic induction vector, $E_{z}$ is the component of the electric field strength vector, $A=\frac{\sigma_{0} B E h_{0}^{2}}{\mu_{0} u^{*}}$ is the magnitude caused by presence of an electric field, $K=\frac{2 \mu_{0} u^{*} l}{h_{0} L^{\prime}}$ is the parameter due to the melt and the rate of dissipation of mechanical energy, $L^{\prime}$ is the specific heat of fusion per unit volume.

At that, the values $\bar{B}=\left\{0 ; B_{y} ; 0\right\}$ and $\bar{E}=\left\{0 ; 0 ; E_{z}\right\}$ are assumed to be given and satisfy the Maxwell equations:

$$
\operatorname{div} \bar{B}=0, \text { rot } \bar{E}=0
$$


Values $\bar{B}, \bar{E}$ and the flow velocity of the electrically conductive lubricant are such that the magnitude of the current strength can be neglected on the electric and magnetic fields.

In a Cartesian coordinate system $x^{\prime} o y^{\prime}$ the equation of the contour of the slider and the molten surface of the guide can be written in the form.

$$
y^{\prime}=h_{0}+x^{\prime} \operatorname{tg} \alpha^{*}, \quad y^{\prime}=-\Phi\left(x^{\prime}\right),
$$

where $\alpha^{*}$ is the angle of slope to the axis $O x^{\prime}$.

The boundary conditions in this case are written in the form:

$$
\begin{gathered}
u=0, \quad v=0 \quad \text { at } \quad y=1+\eta x=h(x), \\
u=0, \quad v=-1 \quad \text { at } y=-\Phi(x), \\
\Phi(x)=\tilde{g}_{0}=K g_{0}=0 \quad \text { at } \quad x=0, \\
p(0)=p(1)=\frac{p_{a}}{p^{*}},
\end{gathered}
$$

where $\eta=\frac{\operatorname{ltg} \alpha^{*}}{h_{0}}$.

The relations between dimensionless and dimensional quantities are given in the form:

$$
\begin{gathered}
u^{\prime}=u^{*} u, \quad v^{\prime}=u^{*} \varepsilon v, \quad x^{\prime}=l x, \quad y^{\prime}=h_{0} y, \\
p^{\prime}=p^{*} p, T^{\prime}=T^{*} T, \beta=T^{*} \beta^{\prime}, \quad \varepsilon=\frac{h_{0}}{l}, \quad \mu^{\prime}=\mu_{0} \mu, \\
\sigma^{\prime}=\sigma_{0} \sigma, \quad \tilde{\alpha}=\frac{\alpha}{p^{*}}, \quad p^{*}=\frac{\mu_{0} u^{*} l}{h_{0}^{2}} .
\end{gathered}
$$

Taking $\mathrm{K}$, caused by the melt and the energy dissipation rate as a small parameter, we seek the function $\Phi(x)$ as:

$$
\Phi(x)=-K \Phi_{1}(x)-K^{2} \Phi_{2}(x)-K^{3} \Phi_{3}(x)-\ldots=H
$$

Boundary conditions for the dimensionless velocity components $u$ and $v$ on the contour $y=0-\Phi(x)$ can be written as:

$$
\begin{aligned}
& v(0-H(x))=v(0)-\left.\left(\frac{\partial v}{\partial y}\right)\right|_{y=0} H(x)-\left.\left(\frac{\partial^{2} v}{\partial y^{2}}\right)\right|_{y=0} H^{2}(x)-\ldots=-1 \\
& u(0-H(x))=u(0)-\left.\left(\frac{\partial u}{\partial y}\right)\right|_{y=0} H(x)-\left.\left(\frac{\partial^{2} u}{\partial y^{2}}\right)\right|_{y=0} H^{2}(x)-\ldots=0 .
\end{aligned}
$$

We seek the asymptotic solution of the system of differential equations (2) with allowance for the boundary conditions (5) and (8) in the form of series in powers of the small parameter K:

$$
\begin{gathered}
v=v_{0}(x, y)+K v_{1}(x, y)+K^{2} v_{2}(x, y)+\ldots, \\
u=u_{0}(x, y)+K u_{1}(x, y)+K^{2} u_{2}(x, y)+\ldots \\
\Phi(x)=-K \Phi_{1}(x)-K^{2} \Phi_{2}(x)-K^{3} \Phi_{3}(x)-\ldots, \\
p=p_{0}(x)+K p_{1}(x)+K^{2} p_{2}(x)+K^{3} p_{3}(x) \ldots \\
T=T_{0}(x)+K T_{1}(x)+K^{2} T_{2}(x)+K^{3} T_{3}(x) \ldots, \\
\mu=\mu_{0}(x)+K \mu_{1}(x)+K^{2} \mu_{2}(x)+K^{3} \mu_{3}(x) \ldots
\end{gathered}
$$

Adding (9) into the system of differential equations (2), taking into account the boundary conditions (5), we obtain the following equations:

- for the zero approximation:

$$
\frac{\partial^{2} v_{0}}{\partial y^{2}}-N v_{0}+A=\frac{1}{j \mu_{0}(x)} \frac{d p_{0}}{d x}, \frac{\partial v_{0}}{\partial x}+\frac{\partial u_{0}}{\partial y}=0
$$

With boundary conditions:

$$
\begin{gathered}
v_{0}=0, \quad u_{0}=0, \quad \text { at } \quad y=1+\eta x, \\
v_{0}=-1, \quad u_{0}=0 \quad \text { at } \quad y=0,
\end{gathered}
$$


- for the first approximation:

$$
\begin{gathered}
\frac{\partial^{2} v_{1}}{\partial y^{2}}=-\frac{\mu_{1}(x)}{\mathrm{j} \mu_{0}^{2}(x)} \frac{d p_{0}}{d x}+\frac{1}{\mathrm{j} \mu_{0}(x)} \frac{d p_{1}}{d x}, \frac{\partial v_{1}}{\partial x}+\frac{\partial u_{1}}{\partial y}=0, \\
\frac{1}{\mathrm{j} \mu_{0}(x)} \frac{d \Phi_{1}(x)}{d x}=\int_{0}^{1+\eta x}\left(\frac{\partial v_{0}}{\partial y}\right)^{2} d y,
\end{gathered}
$$

With boundary conditions:

$$
\begin{gathered}
v_{1}=\left.\left(\frac{\partial v_{0}}{\partial y}\right)\right|_{y=0} \cdot \Phi_{1}(x), \quad u_{1}=\left.\left(\frac{\partial u_{0}}{\partial y}\right)\right|_{y=0} \cdot \Phi_{1}(x) \\
v_{1}=0, \quad u_{1}=0 \quad \text { at } \quad h(x)=1+\eta x, \quad(13) \\
p_{1}(0)=p_{1}(1)=0, \quad K \Phi_{1}(0)=K \tilde{\alpha}, \quad \Phi_{1}(0)=\Phi_{1}(1)=\tilde{\alpha} .
\end{gathered}
$$

Replacing in the first equation of system (2) the velocity $\mathrm{V}$ by its maximum value equal to -1 , the exact solution of the problem for the zero approximation will be sought:

$$
\begin{gathered}
u_{0}=-\frac{\partial \psi_{0}}{\partial x}+U_{0}(x, y), \quad v_{0}=\frac{\partial \psi_{0}}{\partial y}+V_{0}(x, y), \\
\psi_{0}(x, y)=\tilde{\psi}_{0}(\xi), \quad \xi=\frac{y}{h(x)}, \\
V_{0}(x, y)=\tilde{v}(\xi), \quad U_{0}(x, y)=-\tilde{u}_{0}(\xi) \cdot h^{\prime}(x) .
\end{gathered}
$$

Adding (14) into the system of differential equations (10), taking into account the boundary conditions (11), we obtain the following system of differential equations:

$$
\begin{aligned}
& \tilde{\Psi}_{0}^{\prime \prime \prime}=\tilde{C}_{2}, \quad \tilde{v}_{0}^{\prime \prime}=\tilde{C}_{1}, \quad \tilde{u}_{0}^{\prime}+\xi \tilde{v}_{0}^{\prime}=0, \\
& \frac{1}{\mathrm{j} \mu_{0}(x)} \frac{d p_{0}}{d x}=\frac{\tilde{C}_{1}}{h^{2}(x)}+\frac{\tilde{C}_{2}}{h^{3}(x)}+N+A
\end{aligned}
$$

And boundary conditions:

$$
\tilde{\psi}_{0}^{\prime}(0)=0, \quad \tilde{\psi}_{0}^{\prime}(1)=0, \quad \tilde{u}_{0}(1)=0, \quad \tilde{v}_{0}(1)=0
$$

$$
\tilde{u}_{0}(0)=0, \quad \tilde{v}_{0}(0)=-1, \quad \int_{0}^{1} \tilde{v}_{0}(\xi) d \xi=0, \quad p_{0}(0)=p_{0}(1)=\frac{p_{a}}{p^{*}} .
$$

By the direct integration we obtain:

$$
\begin{gathered}
\tilde{\Psi}_{0}^{\prime}(\xi)=\frac{\tilde{C}_{2}}{2}\left(\xi^{2}-\xi\right), \tilde{v}_{0}(\xi)=\tilde{C}_{1} \frac{\xi^{2}}{2}-\left(1+\frac{\tilde{C}_{1}}{2}\right) \xi+1 \\
\tilde{\mathrm{C}}_{1}=6
\end{gathered}
$$

From the condition $p_{0}(0)=p_{0}(1)=\frac{p_{a}}{p^{*}}$ with an accuracy up to the terms of the second order of smallness $O\left(\eta^{2}\right)$ for $\tilde{C}_{2}$ we obtain the expression:

$$
\tilde{C}_{2}=-6\left(1+\frac{1}{2} \eta\right)-(N+A)\left(1+\frac{3}{2} \eta\right)
$$

\section{DeFinition OF Hydrodynamic PRESSURE}

The dimensionless hydrodynamic pressure in the lubricating layer is determined from equation:

$$
\frac{1}{\mathrm{j} \mu_{0}(x)} \frac{d p_{0}}{d x}=\frac{\tilde{C}_{1}}{h^{2}(x)}+\frac{\tilde{C}_{2}}{h^{3}(x)}+N+A
$$

To solve equations (19), we first define $\mu_{0}(x)$. For this, differentiating $\mu_{0}(x)=e^{\alpha p_{0}-\beta T_{0}}$ we obtain:

$$
\frac{d \mu_{0}(x)}{d x}=\mu_{0}(x)\left(\alpha \frac{d p_{0}}{d x}-\beta \frac{d T_{0}}{d x}\right)
$$

In order to define $\frac{d T_{0}}{d x}$ we use the formula for the energy dissipation rate:

$$
\frac{d T_{0}}{d x}=-\frac{24 \mu_{0} \mu_{0}(x) u^{*} l h(x)}{T^{*} c_{p} h_{0}^{2} \tilde{C}_{2}} \int_{0}^{1}\left(\frac{\tilde{\Psi}_{0}^{\prime \prime}(\xi)}{h^{2}(x)}+\frac{\tilde{v}_{0}^{\prime}(\xi)}{h(x)}\right)^{2} d \xi .
$$

Adding (21) into (20) and making a series of transformations, we obtain: 


$$
\begin{aligned}
& \frac{1}{\mu_{0}^{2}(x)} \frac{d \mu_{0}(x)}{d x}=\frac{\alpha \tilde{C}_{1}}{h^{2}(x)}+\frac{\alpha \tilde{C}_{2}}{h^{3}(x)}+ \\
& +\frac{24 \mu_{0} u^{*} l \beta h(x)}{T^{*} c_{p} h_{0}^{2} \tilde{C}_{2}} \int_{0}^{1}\left(\frac{\tilde{\Psi}_{0}^{\prime \prime}(\xi)}{h^{2}(x)}+\frac{\tilde{v}_{0}^{\prime}(\xi)}{h(x)}\right)^{2} d \xi,
\end{aligned}
$$

where $c_{p}$ is heat capacity at constant pressure.

Integrating (22), we obtain:

$\frac{1}{\mu_{0}(x)}=1-\alpha\left[\tilde{C}_{1} J_{2}(x)+\tilde{C}_{2} J_{3}(x)\right]-\frac{D}{\tilde{C}_{2}}\left[\Delta_{1} J_{3}(x)+\Delta_{2} J_{2}(x)+\Delta_{3} J_{1}(x)\right]$,

where $D=\frac{24 \mu_{0} \beta u^{*} l}{T^{*} c_{p} h_{0}^{2}} ; \quad \Delta_{1}=\int_{0}^{1}\left(\tilde{\Psi}_{0}^{\prime \prime}(\xi)\right)^{2} d \xi=\frac{\tilde{C}_{2}^{2}}{12} ;$

$$
\begin{gathered}
\Delta_{2}=2 \int_{0}^{1}\left(\tilde{\Psi}_{0}^{\prime \prime}(\xi) \cdot \tilde{v}_{0}^{\prime}(\xi)\right) d \xi=-\frac{1}{6} \tilde{C}_{1} \tilde{C}_{2} \\
\Delta_{3}=\int_{0}^{1}\left(\tilde{v}_{0}^{\prime}(\xi)\right)^{2} d \xi=4 ; \quad J_{k}(x)=\int_{0}^{x} \frac{d x}{h^{k}(x)} .
\end{gathered}
$$

Substitute the function $\mu_{0}(x)$ by its averaged integral value:

$\tilde{\mu}_{0}=\int_{0}^{1} \mu_{0}(x) d x=$

$=\int_{0}^{1} \frac{d x}{1-\alpha\left[\tilde{C}_{1} J_{2}(x)+\tilde{C}_{2} J_{3}(x)\right]-\tilde{D}\left[\Delta_{1} J_{3}(x)+\Delta_{2} J_{2}(x)+\Delta_{3} J_{1}(x)\right]}$.

Solving the obtained equations $\Delta_{1}$, $\Delta_{2}, \Delta_{3}, J_{3}(x), J_{2}(x), J_{1}(x)$ within an accuracy of $O\left(\eta^{2}\right)$ for $\tilde{\mu}$ obtain the following expression:

$$
\begin{aligned}
& \tilde{\mu}_{0}=1+D \beta\left[\eta\left[50+\frac{26}{3}(N+A)+\frac{29}{6}(N+A)^{2}-\frac{1}{4}(N+A)^{3}\right]-\right. \\
& \left.-\left[102+50(N+A)+\frac{17}{2}(N+A)^{2}+\frac{1}{2}(N+A)^{3}\right]\right]+ \\
& +\alpha\left(6+\frac{(N+A)}{2}-\eta\left(\frac{7}{2}-\frac{(N+A)}{4}\right)\right) .
\end{aligned}
$$

Thus,

$$
p_{0}=j \tilde{\mu}_{0} 3 \eta\left(1+\frac{(N+A)}{2}\right)\left(x^{2}-x\right)+\frac{p_{a}}{p^{*}}
$$

In order to define $\Phi_{1}(\theta)$ taking into account equations (17) and (25), we come to the following equation:

$$
\frac{d \Phi_{1}}{d x}=j \tilde{\mu}_{0} h(x) \int_{0}^{1}\left(\frac{\psi_{0}^{\prime \prime}(\xi)}{h^{2}(x)}+\frac{\tilde{v}_{0}^{\prime}(\xi)}{h(x)}\right)^{2} d \xi
$$

Integrating equation (27), we obtain:

$$
\Phi_{1}(x)=j \tilde{\mu}_{0}\left(\int_{0}^{x} \frac{\Delta_{1} d x}{h^{3}(x)}+\int_{0}^{x} \frac{\Delta_{2} d x}{h^{2}(x)}+\int_{0}^{x} \frac{\Delta_{3} d x}{h(x)}\right)
$$

Solving the equation (28) at the condition $K \Phi_{1}(0)=K \tilde{\alpha}$, we obtain:

$$
\Phi_{1}(x)=j \tilde{\mu}_{0}\left[\frac{\tilde{C}_{2}^{2}}{12}\left(x-\frac{3}{2} \eta x^{2}\right)+\tilde{C}_{2}\left(x-\eta x^{2}\right)+4\left(x-\frac{1}{2} \eta x^{2}\right)+\tilde{\alpha}\right] .
$$

The exact self-similar solution for the first approximation will be sought in the form:

$$
\begin{gathered}
u_{1}=-\frac{\partial \psi_{1}}{\partial x}+U_{1}(x, y), \quad v_{1}=\frac{\partial \psi_{1}}{\partial y}+V_{1}(x, y), \\
\psi_{1}(x, y)=\tilde{\psi}_{1}(\xi), \quad \xi=\frac{y}{h(x)}, \\
V_{1}(x, y)=\tilde{v}(\xi), \quad U_{1}(x, y)=-\tilde{u}_{1}(\xi) \cdot h^{\prime}(x) .
\end{gathered}
$$

Adding (30) into the system of differential equations (12), taking into account the boundary conditions (13), we obtain the following system of differential equations:

$$
\begin{gathered}
\tilde{\Psi}_{1}^{\prime \prime \prime}(\xi)=\tilde{\tilde{C}}_{2}, \quad \tilde{v}_{1}^{\prime \prime}=\tilde{\tilde{C}}_{1}, \quad \tilde{u}_{1}^{\prime}+\xi \tilde{v}_{1}^{\prime}=0, \\
\frac{1}{\mathrm{j} \tilde{\mu}_{0}} \frac{d p_{1}}{d x}-\frac{\mu_{1}(x)}{\mathrm{j} \tilde{\mu}_{0}^{2}} \frac{d p_{0}}{d x}=\frac{\tilde{\tilde{C}}_{1}}{h^{2}(x)}+\frac{\tilde{\tilde{C}}_{2}}{h^{3}(x)} .
\end{gathered}
$$

And boundary conditions:

$$
\tilde{\Psi}_{1}^{\prime}(0)=0, \quad \tilde{\Psi}_{1}^{\prime}(1)=0, \quad \tilde{u}_{1}(1)=0, \quad \tilde{v}_{1}(1)=0
$$


$\tilde{v}_{1}(0)=M, \tilde{u}_{1}(0)=0, \int_{0}^{1} \tilde{v}_{1}(\xi) d \xi=0, p_{1}(0)=p_{1}(1)=0$

By the direct integration we obtain:

$$
\begin{gathered}
\tilde{\Psi}_{1}^{\prime}(\xi)=\frac{\tilde{\tilde{C}}_{2}}{2}\left(\xi^{2}-\xi\right), \quad \tilde{v}_{1}(\xi)=\tilde{\tilde{C}}_{1} \frac{\xi^{2}}{2}-\left(\frac{\tilde{\tilde{C}}_{1}}{2}+M\right) \xi+M, \\
\tilde{\tilde{C}}_{1}=6 M
\end{gathered}
$$

where

$$
\begin{aligned}
& M=\left.\sup _{x \in[0 ; 1]}\left(\frac{\partial v_{0}}{\partial y}\right)\right|_{y=0} \cdot \Phi_{1}(x)= \\
& =\sup _{x \in[0 ; 1]}\left[1-\eta x-\frac{3}{2 \tilde{\mu}_{0}} \eta(2 x-1)\left(1+\frac{N+A}{2}\right)-\frac{N+A}{2}(1+\eta x)\right] \times \\
& \times j \tilde{\mu}_{0}\left\{\frac{\tilde{C}_{2}^{2}}{12}\left(x-\frac{3}{2} \eta x^{2}\right)+\tilde{C}_{2}\left(x-\eta x^{2}\right)+4\left(x-\frac{1}{2} \eta x^{2}\right)+\tilde{\alpha}\right\} \mid .
\end{aligned}
$$

In order to find the value $\tilde{\tilde{C}}_{2}$ and to solve the equation for hydrodynamic pressure:

$$
\frac{1}{\mathrm{j} \tilde{\mu}_{0}} \frac{d p_{1}}{d x}-\frac{\mu_{1}(x)}{\mathrm{j} \tilde{\mu}_{0}^{2}} \frac{d p_{0}}{d x}=\frac{\tilde{\tilde{C}}_{1}}{h^{2}(x)}+\frac{\tilde{\tilde{C}}_{2}}{h^{3}(x)},
$$

we define $\mu_{1}(x)$ first. For this we differentiate the expression $\mu_{1}(x)=e^{\alpha p_{1}-\beta T_{1}}:$

$\frac{d \mu_{1}(x)}{d x}=\alpha \mu_{0}(x) \frac{d p_{1}}{d x}+\alpha \mu_{1}(x) \frac{d p_{0}}{d x}-\beta \mu_{1}(x) \frac{d T_{0}}{d x}-\beta \mu_{0}(x) \frac{d T_{1}}{d x}$

In order to define $\frac{d T_{1}}{d x}$ we use the formula for the energy dissipation rate:

$\frac{d T_{1}}{d x}=\frac{24 \mu_{0} \mu_{1}(x) u^{*} \ln (x)}{T^{*} c_{p} h_{0}^{2} \tilde{\tilde{C}}_{2}} \int_{0}^{1} 2\left(\frac{\tilde{\Psi}_{0}^{\prime \prime}(\xi)}{h^{2}(x)}+\frac{\tilde{v}_{0}^{\prime}(\xi)}{h(x)}\right)\left(\frac{\tilde{\Psi}_{1}^{\prime \prime}(\xi)}{h^{2}(x)}+\frac{\tilde{v}_{1}^{\prime}(\xi)}{h(x)}\right) d \xi$.

Adding (35) to (34) and making a series of transformations up to terms $O(K \alpha)$, we obtain:

$\frac{1}{\mu_{1}(x)}=1-2 \frac{D}{\tilde{C}_{2}}\left[\tilde{\Delta}_{1} \tilde{J}_{3}(x)+\tilde{\Delta}_{2} \tilde{J}_{2}(x)+\tilde{\Delta}_{3} \tilde{J}_{2}(x)+\tilde{\Delta}_{4} \tilde{J}_{1}(x)\right]$,

where $\tilde{\Delta}_{1}=\int_{0}^{1} \tilde{\Psi}_{0}^{\prime \prime}(\xi) \tilde{\Psi}_{1}^{\prime \prime}(\xi) d \xi ; \quad \tilde{\Delta}_{2}=\int_{0}^{1} \tilde{\Psi}_{0}^{\prime \prime}(\xi) \cdot \tilde{v}_{1}^{\prime}(\xi) d \xi$

$$
\begin{gathered}
\tilde{\Delta}_{3}=\int_{0}^{1} \tilde{\Psi}_{1}^{\prime \prime}(\xi) \cdot \tilde{v}_{0}^{\prime}(\xi) d \xi ; \quad \tilde{\Delta}_{4}=\int_{0}^{1} \tilde{v}_{0}^{\prime}(\xi) \cdot \tilde{v}_{1}^{\prime}(\xi) d \xi \\
\tilde{J}_{k}(x)=\int_{0}^{x} \frac{d x}{h^{k}(x)} .
\end{gathered}
$$

Substitute the function $\mu_{1}(x)$ by its averaged integral value:

$$
\begin{aligned}
& \tilde{\mu}_{1}=1+D \beta\left\{-\frac{1}{2}+\frac{5 \eta}{12}+\frac{N+A}{6}\left(\frac{1}{2}+\frac{\eta}{4}\right)+\right. \\
& \left.+\frac{M}{\tilde{\tilde{C}}_{2}}\left[\left(10-\frac{\eta}{3}\right)+(N+A)\left(1+\frac{5 \eta}{6}\right)\right]\right\} .
\end{aligned}
$$

Then for $p_{1}$ we obtain:

$$
\begin{aligned}
& p_{1}=j \tilde{\mu}_{0}\left[6 M\left(x-\eta x^{2}\right)+\tilde{\tilde{C}}_{2}\left(x-\frac{3}{2} \eta x^{2}\right)\right]+ \\
& +3 \eta \mathrm{j} \tilde{\mu}_{1}\left(1+\frac{N+A}{2}\right)\left(x^{2}-x\right) .
\end{aligned}
$$

From the condition $p_{1}(0)=p_{1}(1)=0$ we obtain:

$$
\tilde{\tilde{C}}_{2}=-6 M\left(1+\frac{\eta}{2}\right)
$$

Adding $\tilde{\tilde{C}}_{2}$ to (37) for $\tilde{\mu}_{1}$ we eventually obtain:

$$
\begin{aligned}
& \tilde{\mu}_{1}=1+D \beta\left\{-\frac{1}{2}+\frac{5 \eta}{12}+\frac{N+A}{6}\left(\frac{1}{2}+\frac{\eta}{4}\right)-\right. \\
& \left.-\frac{1}{3}\left(5-\frac{8}{3} \eta+(N+A)\left(\frac{1}{2}+\frac{\eta}{6}\right)\right)\right\},
\end{aligned}
$$

\section{RESULTS OF RESEARCH AND THEIR DISCUSSION}

For the bearing capacity and the frictional force, we obtain: 


$$
\begin{aligned}
& W=\frac{\mu_{0} l^{2} u^{*}}{h_{0}^{2}} \int_{0}^{1}\left(p_{0}-\frac{p_{a}}{p^{*}}+K p_{l}\right) d x=\frac{\mu_{0} l^{2} u^{*} j}{h_{0}^{2}}\left[-\frac{\eta \tilde{\mu}_{0}}{2}\left(1+\frac{N+A}{2}\right)+\right. \\
& \left.+K\left\{\tilde{\mu}_{0}\left[6 M\left(\frac{1}{2}-\frac{\eta}{3}\right)-6 M \tilde{\mu}_{0}\left(\frac{1}{2}-\frac{\eta}{4}\right)\right]-\frac{\eta \tilde{\mu}_{1}}{2}\left(1+\frac{N+A}{2}\right)\right\}\right]
\end{aligned}
$$

$L_{\text {тр }}=\frac{\mu_{0} l^{2} u^{*}}{h_{0}} \int_{0}^{1}\left[\left.\frac{\partial v_{0}}{\partial y}\right|_{y=0}+\left.K \frac{\partial v_{1}}{\partial y}\right|_{y=0}\right] d x=$

$=\frac{\mu_{0} l^{2} u^{*}}{h_{0}}\left[\tilde{\mu}_{0} \mathrm{j}\left(1-\frac{\eta}{2}-\frac{(N+A)}{2}\left(1+\frac{\eta}{2}\right)\right)+K M\left(4-5 \eta-3 \tilde{\mu}_{0}\left(1-\frac{3 \eta}{2}\right)\right)\right]$.

For numerical analysis, the following range of parameter changes has been used:

$$
\begin{gathered}
\mu_{0}=0.001022 \mathrm{Hs} / \mathrm{m}^{2}, \eta=0.3 \ldots 1 \mathrm{~m}, l=0.1256 \ldots 0.1884 \mathrm{~m}, \\
u^{*}=1 . .3 \mathrm{~m} / \mathrm{s}, h_{0}=10^{-7} \ldots 2 \cdot 10^{-6} \mathrm{~m}, \\
K=0.0000022 \ldots 0.00052, M=6,47 \ldots 66,44 .
\end{gathered}
$$$$
p_{a}=0.08 \div 0.101325 \mathrm{MПа}, \alpha=0 \ldots 1, L^{\prime}=35,33 \ldots 38,1 \mathrm{H} / \mathrm{m}^{2} \text {. }
$$

Based on the results of numerical calculations (the average values of the range of variation were used to plot the graphs), the graphs shown in Fig. 2-3 were plotted.

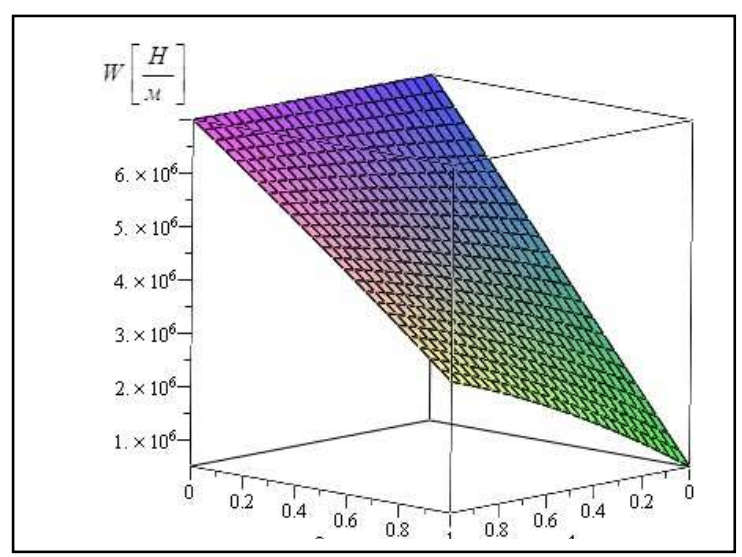

Fig. 2. Dependence of the bearing capacity on the parameter A, caused by the presence of an electric field, and the parameter $\beta$, characterizing the dependence of the viscosity of the lubricant on temperature

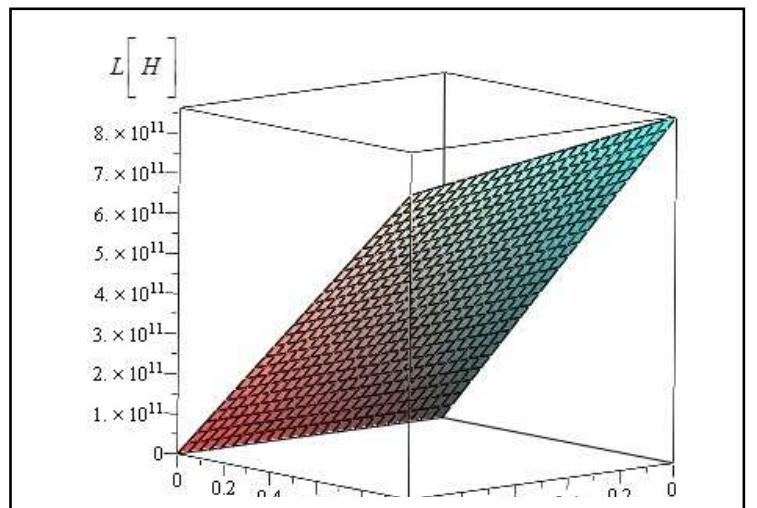

Fig. 3. Dependence of the friction force A, caused by the presence of an electric field, and the parameter $\beta$, characterizing the dependence of the viscosity of the lubricant on temperature.
1. A refined design model of a thrust sliding bearing working under conditions of hydrodynamic lubrication with a melt of a low-melting coating is obtained, taking into account the rheological properties of the electrically conductive lubricant, and also taking into account the dependence of the viscosity and electrical conductivity of the lubricant on pressure and temperature.

2. A significant contribution of the design parameter $A$ caused by the presence of an electric field, the parameter $\mathrm{K}$ characterizing the rate of dissipation of mechanical energy, the parameter $\beta$, characterizing the dependence of the viscosity of the lubricant on temperature, and on the Hartmann number $N$. As the parameters $\mathrm{K}, \mathrm{A}, \mathrm{N}$ increase, the coefficient of friction decreases by $28 \%$, and the bearing capacity increases by $12 \%$.

\section{CONCLUSION}

The obtained results in the form of design models can be used in the development and carrying out of verification calculations of the structure of sliding supports operating on electrically conductive lubricants.

\section{Acknowledgment}

The work was has been made under the grant of Russian Railways No. 2210370/22.12.2016 for the development of scientific and pedagogical schools in the field of railway transport.

\section{References}

[1] D.Y. Kropachev, A.A. Grishin, A.D. Maslo, "Ways of Expeditious Measurement of Temperature of Fusion of Metals for Needs of Machine-Building Enterprises," Casting and Metallurgy, No. 3(66), pp. 126-127, 2012.

[2] Wilson, "Lubricant with Fusion," Problems of Friction and Lubricant, No. 1, pp. 19, 1976.

[3] Beretta, Niro, Silvestri, "The Sliding Bearings Greased by Own Fusion or a Product of Sublimation," The Works Amer. islands ing. - fur, No. 1 , pp. 86-90, 1992.

[4] Physical Quantities, Reference book, Energoatomizdat, 1991.

[5] V.Y. Havin, Short Chemical Reference Book, L.: Chemistry, 1991.

[6] V.I. Perelman, Short Reference Book of the Chemist, M.-L.: Chemistry, 1964.

[7] Reference Book on Soldering, Prod. the 2nd, updated and revised, M.: Mechanical Engineering, 1984.

[8] L.I. Kotelnitskaya, N.N. Demidova, "Calculation Radial with Effective Work on Lubricant with Fusion in the Turbulent Mode," Vestnik RGUPS, No. 2, pp. 18-23, 2002.

[9] V.M. Prikhodko, L.I. Kotelnitskaya, "Mathematical Model of Hydrodynamic Lubricant When Melting a Basic Surface of the Radial Bearing," Friction and Wear,V. 22, No. 6, pp. 606-608, 2001.

[10] E.A. Zadorozhnaya, I.V. Mukhortov, I.G. Levanov, "Application of Non-Newtonian Models of Lubricant Fluids at Calculation ComplexLoaded Units of Friction Piston and Rotor-Type Machines," Friction and Lubricant in Machines And Mechanisms, No. 7, pp. 22 - 3, 20110.

[11] V.N. Prokopiev, A.K. Boyarshinova, E.A. Zadorojnaya, "Dynamics of the Complex-Loaded Bearing Greased with Non-Newtonian Liquid," Problem of Mechanical Engineering and Reliability of Machines, No. 6, pp. $108-114,2005$.

[12] V.N. Prokopiev, E.A. Zadorozhnaya, V.G. Karavaev, I.G. Levanov, "Improvement of the Computation Procedure for Complex-Loaded 
Sleeve Bearings Lubricated with Non-Newtonian Oils," Journal of Machinery Manufacture and Reliability, № 1, pp. 63- 67, 2010.

[13] K.S. Akhverdiev, M.A. Mukutadze, E.O. Lagunova, K.S. Solop, "Working Out of an Analytical Model of a Radial Bearing Taking into Account Dependence of Viscous Characteristics of Micropolar Lubrication on Pressure and Temperature," International Journal of Applied Engineering Research, Vol. 12, Number 15, pp. 4840-4846, 2017.

[14] E.O. Lagunova, "Simulation Model of Radial Bearing, Taking into Account the Dependence of Viscosity Characteristics of Micro-Polar Lubricant Material on Temperature," International Journal of Applied Engineering Research, Vol. 12, Number 12, pp. 3346-3352, 2017.

[15] E.O. Lagunova, "Design Model of Radial Bearing Taking into Account the Dependence of the Viscosity of Lubricant on Pressure and Temperature," Global Journal of Pure and Applied Mathematics, Vol. 13, Number 7, pp. 3531-3542, 2017.

[16] K.S. Akhverdiev, M.A. Mukutadze, A.C. Erkenov Development of Systems of Settlement Models of Sliding Bearings on the Basis of Improvement of the Elastodynamic Theory Of Lubricant: Monograph Rostov n/D. RSTU, 2012.

[17] K.S. Akhverdiev, M.A. Mukutadze, E.O. Lagunova, V.V. Vasilenko, "Hydrodynamic Calculation of the Radial Bearing with Lubricated Melt Fusible Coating in the Presence of Lubricant," Vestnik RGUPS, №2 (66), pp. 129-135, 2017.

[18] V.V. Vasilenko, E.O. Lagunova, M.A. Mukutadze, "Hydrodynamic Calculation of the Radial Bearing, the Lubricated Melt Coating in the Presence of a Lubricant," Online journal «Naukovedenie», V. 9, №5, 2017
[19] K.S. Akhverdiev, E.O. Lagunova, V.V. Vasilenko, "Design Model of Radial Melt-Lubricated Bearing Taking into Account Pressure-Viscosity Ratio," Vestnik of Don State Technical University, vol. 17, no.3, pp. 2737, 2017.

[20] E.O. Lagunova, "Wedge-Shaped Sliding Supports Operating on Viscoelastic Lubricant Material Caused by the Melt, Taking Into Account the Dependence of Viscosity and Shear Modulus on Pressure," International Journal of Applied Engineering Research, 2017, Vol. 12, Number 19, pp. 9120-9127.

[21] E.O. Lagunova, "Radial Plain Bearings Operating on Viscoelastic Lubricant Caused by the Melt, Taking into Account the Dependence of the Viscosity of the Lubricant and the Shear Modulus on the Pressure," International Journal of Applied Engineering Research, Vol. 12, Number 19, pp. 9128-9137, 2017.

[22] V.V. Vasilenko, E.O. Lagunova, M.A. Mukutadze, V.M. Prikhodko, "Calculation Model of the Radial Bearing, Caused by the Melt, Taking into Account the Dependence of Viscosity on Pressure," International Journal of Applied Engineering Research, Vol. 12, Number 19, pp. 9138-9148, 2017.

[23] K.S. Akhverdiev, M.A. Mukutadze, E.O. Lagunova, V.V. Vasilenko, "The Wedge-Shaped Sliding Support Using Micropolar Lubricant, Caused by Fusion," Vestnik RGUPS, No. 3 (67), pp. 8-15, 2017.

[24] E.O. Lagunova, M.A. Mukutadze, K.S. Solop, "Working Out of an Analytical Model of an Axial Bearing Taking into Account Dependence of Viscous Characteristics of Micropolar Lubrication on Pressure and Temperature," International Journal of Applied Engineering Research, Vol. 12, Number 14, pp. 4644-4650, 2017. 